\title{
3-Gyr-old Globular Clusters in the Merger Remnant NGC 1316: Implications for the Fate of Globular Clusters Formed During Gas-rich Galaxy Mergers
}

\author{
Paul Goudfrooij \\ Space Telescope Science Institute, 3700 San Martin Drive, Baltimore, \\ $M D$ 21218, U.S.A.
}

\begin{abstract}
The giant early-type merger remnant galaxy NGC 1316 is an ideal probe for studying the long-term effects of a major merger on its globular cluster (GC) system, given its spectroscopically derived merger age of $\sim 3 \mathrm{Gyr}$ which we reported in a recent paper. Here we report several pieces of photometric evidence showing that the second-generation GCs in NGC 1316 are at an evolutionary phase in between that of luminous GCs found in younger merger remnants such as (e.g.) NGC 7252 and that of 'red' GCs found in 'normal, old' ellipticals. The observation that massive, second-generation GCs formed during major mergers can survive for at least 3 Gyr provides strong evidence that these clusters can have 'normal' mass functions including low-mass stars, and hence that they can survive to reach 'old age' similar to those of 'normal' ellipticals.
\end{abstract}

\section{Introduction}

As this symposium emphasized once again, the study of globular clusters (GCs) around galaxies provides unique and important information regarding our understanding of how and when galaxies form. Their nature as simple stellar population (SSP) significantly simplifies the determination of their ages and metallicities relative to that of stellar populations that constitute the integrated light of their parent galaxies. A particularly interesting and now well-known feature of the GC systems of giant early-type galaxies is the presence of bimodal color distributions, providing obvious evidence for the occurrence of a 'second event' during the formation history of these galaxies (as discussed by many authors in this volume [e.g., Brodie, Kissler-Patig, Schweizer, Zepf]). To date, the only 'simple' model that actually predicted such a bimodality was that of Ashman \& Zepf (1992), which modeled the properties of GCs forming in a major merger of gas-rich spirals (together with the giant elliptical host galaxies themselves). They figured that the enriched gas associated with the spiral disks would form 'red' (i.e., metal-rich) GCs during the merger. Soon afterwards, observations of young merger remnant galaxies with the Hubble Space Telescope (HST) revealed the presence of luminous young GCs (e.g., Holtzman et al. 1992; Whitmore et al. 1993), further supporting this 'merger scenario'.

However, certain properties of the GC systems around some giant ellipticals (e.g., very large GC specific frequencies of central cluster galaxies) do not seem 
to fit into this simple merger scenario, which prompted several groups to propose alternative concepts to explain the GC color bimodality. One such concept is 'multi-phase collapse' (Forbes, Brodie, \& Grillmair 1997) which proposes that the metal-rich GCs are formed during a 'secondary collapse' phase of the galaxy which does not involve a merger. Another popular concept is the 'accretion scenario' (Côté, Marzke \& West 1998) which proposes that every galaxy is born with a GC system that has a median color according to the color-magnitude relation among galaxies. The color bimodality in giant ellipticals then arises through accretion of small galaxies by a large galaxy.

Given these different points of view, it is important to obtain additional evidence for or against the 'merger scenario'. Two rather major sources of debate in this regard are: (i) Whether or not the GCs formed in mergers lack low-mass stars so that they will disappear before reaching old age (see, e.g., Brodie et al. 1998; Smith \& Gallagher 2001), and (ii) whether or not general properties of GCs formed in mergers (i.e., their spatial distributions, ages, metallicities and specific frequencies) are compatible with those of 'red' GCs in old ellipticals. These two issues are addressed in the remainder of this paper, using new results on the GC system of the giant intermediate-age merger remnant galaxy NGC 1316.

\section{Spectroscopic Evidence for 3-Gyr-old GCs in NGC 1316}

We (Goudfrooij et al. 2001, hereafter Paper I) recently published results of multi-slit spectroscopy of GC candidates in NGC 1316, an early-type galaxy that is an obvious merger remnant (e.g., Schweizer 1980). We discovered the presence of $\sim 10$ GCs associated with NGC 1316 that have luminosities up to $\sim 10$ times higher than that of $\omega$ Cen. Our measurements of $\mathrm{H} \alpha$ and the Ca II triplet in the spectra of the brightest GCs showed them to have solar metallicity (to within $0.15 \mathrm{dex}$ ) and to have an age of $3.0 \pm 0.5$ Gyr (Paper I; also shown as Fig. 2b in Schweizer's contribution to this volume). These are obviously GCs that have formed from enriched gas during a merger. This reinforces the view that luminous GCs formed during mergers do not necessarily have high low-mass cutoffs to their IMFs, and it also means that they can actually survive dynamical disruption processes taking place during and after the merging process.

\section{Color-Magnitude Diagram of the GC system of NGC 1316}

We have studied the photometric properties of the GC system of NGC 1316 using a combination of $H S T / W F P C 2$ and large-field ground-based photometry using the ESO NTT. The observations will be described in full detail in a forthcoming MNRAS paper; here we only show and discuss relevant results.

The $B$ vs. $B-I$ color-magnitude diagram (CMD) from the WFPC2 data is shown in panel (c) of Fig. 1. The area which the GC system of the halo of the Milky Way (Harris 1996) would occupy if placed at the distance of NGC 1316 is also indicated (see arrow). The positions of GC candidates in this CMD are compared to predictions of SSP synthesis models in panels (a) and (b) of Fig. 1 which show the time evolution of $B-I$ and $M_{B}$ using the SSP models of Bruzual \& Charlot (1996, hereafter BC96). The metallicity range encompassed by the brightest clusters at an age of $3 \pm 0.5 \mathrm{Gyr},-0.15 \lesssim\left[Z / Z_{\odot}\right] \lesssim 0.15$, 


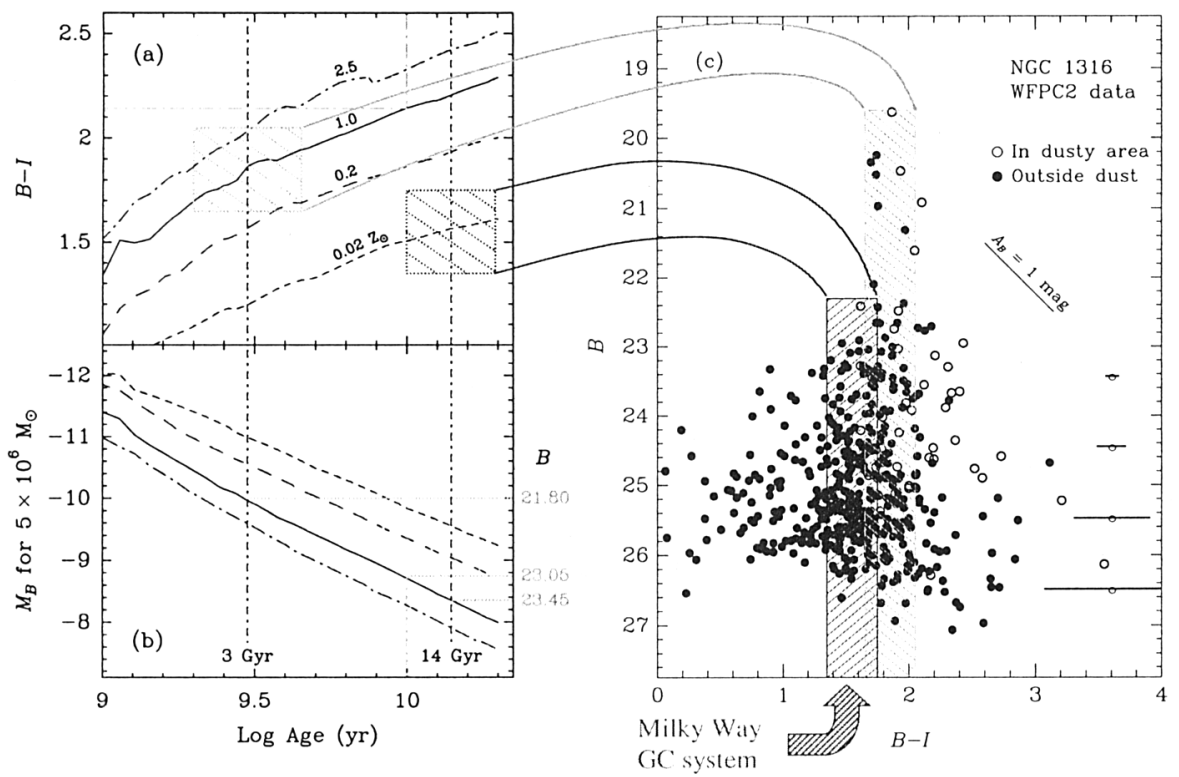

Figure 1. Panel (a): Time evolution of $B-I$ according to the Bruzual \& Charlot (1996) SSP models. Metallicities are indicated above the models. The hatched region on the left indicates the $B-I$ color interval populated by GCs of age $3.0 \pm 0.5$ Gyr and with $\left[\mathrm{Z} / \mathrm{Z}_{\odot}\right]=1.0 \pm 0.15$ dex, while the hatched region on the right does so for a old, metalpoor cluster population such as that of the Milky Way halo. Panel (b): Luminosity evolution for a GC having the mass of $\omega$ Cen. Curves as in Panel (a). $B$ magnitudes (at the distance of NGC 1316) for solar metallicity at 3,10 and $14 \mathrm{Gyr}$ are indicated on the right. Panel (c): $B$ vs. $B-I$ CMD from the $H S T$ photometry. The two hatched regions are the same ones as indicated on Panel (a). Note that the hatched regions fit the observed CMD of NGC 1316 clusters very well.

corresponds to $1.75 \leq B-I \leq 1.95$ according to the BC96 models. Considering the typical photometric errors of the WFPC2 photometry as well, we assigned $1.65 \leq B-I \leq 2.05$ to the putative second-generation population of clusters, indicated as the hatched region on the left side of panel (a). The other hatched region indicates the $B-I$ interval occupied by the Galactic GC system which corresponds to a metallicity range of $-1.7 \lesssim\left[Z / Z_{\odot}\right] \lesssim-0.8$ (Harris 1996), which is consistent with the model predictions at an age of 14 Gyr. Fig. 1 shows that these two intervals provide a very good fit to the positions of the cluster candidates on the CMD. Panel (b) shows that even after luminosity dimming to an age of say $10 \mathrm{Gyr}$, the 'red' clusters are up to several magnitudes more luminous (and hence more massive) than that of the most massive GC in our Galaxy. 
Our conclusion is that we are dealing with the presence of two distinct cluster populations: (i) a second-generation population clusters of roughly solar metallicity that were formed $\sim 3$ Gyr ago during a major spiral-spiral merger, and (ii) a population of old, primarily metal-poor clusters that were associated with the progenitor galaxies.

\section{Radial Distributions of the GC (sub-)populations in NGC 1316}

The radial surface density distribution of GC candidates in NGC 1316 is depicted in Fig. 2a, along with that of the integrated light of the galaxy. The GC surface density is seen to flatten off somewhat towards the centre with respect to the integrated light profile (note that this excludes the possibility of being due to extinction by dust features in the inner regions). This is a known feature of GC systems of 'old' giant ellipticals, in which this flattening is more pronounced (e.g., Forbes et al. 1998). It is most probably due to the accumulative effects of tidal shocking (which is most effective in the central regions) and dynamical friction during the first few dynamical time-scales after the assembly of the galaxy (see, e.g., Fall and Vesperini's contributions to this volume). The more moderate flattening of the surface density of the GC system of NGC 1316 towards the centre relative to those of 'old' ellipticals is consistent with the notion that NGC 1316 is only a few Gyr old. As to the GC surface density profile in the outer regions, fitting a power law results in a slope of $-1.36 \pm 0.10$, which is consistent with the slope for the $B$-band light of the galaxy in the same radial range $(-1.41 \pm 0.02)$. This suggests that the GC system originally experienced the same violent relaxation as did the main body of the galaxy.

A key prediction of the 'merger scenario' of Ashman \& Zepf (1992) was that the metal-rich GCs formed in mergers will be more centrally concentrated than the pre-existing metal-poor GCs, which is the situation in well-studied giant ellipticals (e.g,. Geisler, Lee, \& Kim 1996). NGC 1316 is an important probe to test this prediction, since dynamical evolution has acted over a much longer time scale in NGC 1316 than in merger remnants studied to date (which are younger). To this end, we defined the 'blue' and 'red' subpopulations as follows: $1.00 \leq B-I \leq 1.55$ ('blue') and $1.75 \leq B-I \leq 2.50$ ('red'). This definition purposely avoids the area overlapping the two hatched regions on Fig. 1c. The radial distributions of both subpopulations are depicted on Fig. $2 \mathrm{~b}$. The 'metal-rich' clusters are indeed clearly more centrally concentrated than the 'metal-poor' ones, consistent with the predictions of the 'merger scenario'.

Due to the page limit of this contribution, we refer the reader to a forthcoming MNRAS paper (astro-ph/0107533) which will include other photometric properties of the GC system of NGC 1316 (e.g., luminosity functions for both subpopulations, the specific frequency, and its evolution).

Acknowledgments. It is a pleasure to thank the organizing committee (and Ms. Star Cluster and Doug Geisler in particular), who did a marvelous job in organizing this Symposium. I'd also like to express a big thank you to my excellent collaborators on this project: Vicky Alonso, Markus Kissler-Patig, Jen Mack, Claudia Maraston, Georges Meylan and Dante Minniti. 

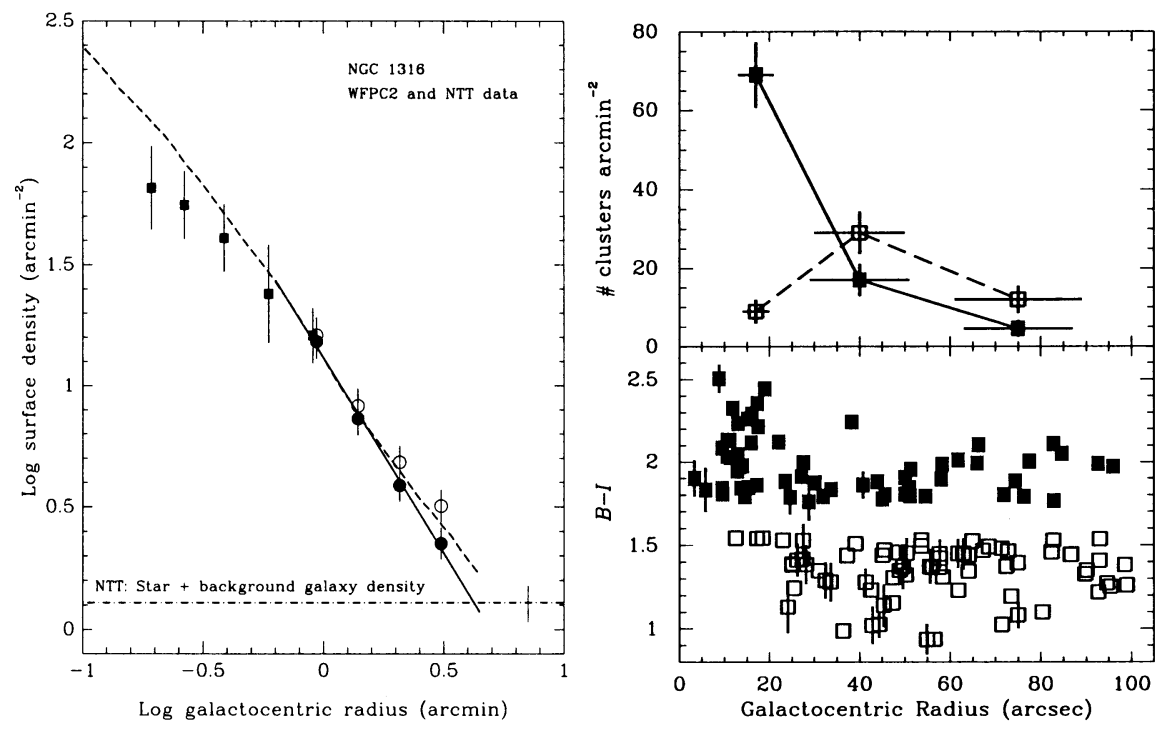

Figure 2. (a, left) Radial distribution of the GC system of NGC 1316 down to $B=24.0$. Filled squares represent measurements from the WFPC2 data, corrected for foreground stars and background galaxies. Circles represent measurements from the ESO NTT data. The open and filled circles represent different corrections for the density of background galaxies. Reality is likely to lie in between the two sets of symbols. A power-law fit to the solid circles beyond the inner 1 arcmin is shown by a solid line. The surface brightness profile of the integrated $B$-band light of the galaxy (normalized to the surface density of star clusters at a radius of $1 \mathrm{arcmin}$ ) is shown by the dashed line. (b, right) Radial distributions of $B-I$ and surface density for the two GC subpopulations from the WFPC2 data. Filled squares represent the 'red' GCs and open squares represent the 'blue' GCs.

\section{References}

Ashman, K. M., \& Zepf, S. E., 1992, ApJ, 384, 50

Brodie, J. P., Schroder, L. L., Huchra, J. P., et al., 1998, AJ, 116, 691

Côté, P., Marzke, R. O., \& West, M. J., 1998, ApJ, 501, 554

Forbes, D. A., Brodie, J. P., \& Grillmair, C. J., 1997, AJ, 113, 1652

Forbes, D. A., Grillmair, C. J., Williger, G. M., et al., 1998, MNRAS, 293, 325

Geisler, D., Lee, M. G., \& Kim, E., 1996, AJ, 111, 1529

Goudfrooij, P., Mack, J., Kissler-Patig, M., et al., 2001, MNRAS, 322, 643

Harris, W. E., 1996, AJ, 112, 1487

Holtzman, J. A., Faber, S. M., Shaya, E. J., et al., 1992, AJ, 103, 691

Schweizer, F., 1980, ApJ, 237, 303

Smith, L. J., \& Gallagher, J. S. III, 2001, MNRAS, in press (astro-ph/0104429) 
Whitmore, B. C., Schweizer, F., Leitherer, C., et al., 1993, AJ, 106, 1354

\section{Discussion}

F. Schweizer: A comment and a question. This is the last evidence yet that I have seen for the existence of intermediate-age globular clusters in an early-type galaxy. The unusual IMF required for the globulars in NGC 1275 observed by Brodie et al (1998) is an artifact, I believe, of their having used the 1993 models of Bruzual \& Charlot to observe the clusters Balmer line strengths. With the 1996 Bruzual \& Charlot models, the problem disappears because these models predict 20\% - 30\% stronger Balmer absorption lines. Hence my question: Did you use relatively new Bruzual \& Charlot models (1996 or 2000) for your age interpretation?

P. Goudfrooij: Yes, I actually used both the 1996 and 2000 versions of the Bruzual \& Charlot models for this study. I found that for the age/metallicity parameter space involved, there is no discernible difference between the two sets of models.

J. Brodie: Our result of a biassed IMF in the NGC 1275 cluster still stands. In Brodie et al 1998 we did indeed use the Bruzual \& Charlot 1993 models for our analysis of the NGC 1275 data but we were aware of the differences between the 1993 and 1996 versions of the model. We took those differences into account in the details of how we defined our indices and manipulated the model spectra before making our comparisons. 